\title{
Some aspects of cancer biomarkers and their clinical application in solid tumors - revisited
}

\author{
Daniel Isaac ${ }^{1}$, Jailan Elayoubi ${ }^{1}$, Abdullah Almohammadi ${ }^{1}$ and Nikolay V. Dimitrov ${ }^{1, *}$ \\ 'Division of Hematology and Oncology, Department of Medicine, College of Human Medicine, Michigan State University, East Lansing, MI, USA
}

\begin{abstract}
Cancer biomarkers can be used for a variety of purposes related to screening, prediction, stratification, detection, diagnosis, prognosis, treatment design, and monitoring of a therapeutic response. One of the most important characteristics of a given biomarker includes ease of collection allowing for a non-invasive approach and frequent sampling. Such samples may be obtained from serum or plasma, sputum, bronchoalveolar lavage, saliva, nipple discharge, pleural, or peritoneal effusions. Validation of different biomarkers is considered a mandatory method for useful evaluation. In this review, we highlight the clinical applicability of some cancer biomarkers, as well as future approaches for their development and collection, which may help guide clinicians and researchers. The role of liquid biopsies will also be summarized. Further studies using liquid biopsies are needed to elucidate the significance of various sources of biomarkers suitable for clinical application.
\end{abstract}

Keywords: biomarkers; liquid biopsy; genetic material; diagnosis; prognosis

\section{Background}

Biomarkers are defined as a biological molecule that can be used to distinguish an abnormal from normal process or condition. In the field of cancer, biomarkers are identified as specific molecules that help to distinguish between normal and cancerous conditions and may potentially enable the development of more effective diagnostic or prognostic tools [1-3]. Cancer biomarkers can consist of biomolecules used for medical purposes such as proteins, lipids, carbohydrates, metabolites, and genetic material (DNAs, methylated DNAs, RNAs, micro RNA). Based on their purpose, biomarkers are generally classified into four categories: diagnostic, prognostic, therapeutic and prediction/staging related.

Evaluating biomarkers that are related to patient's prognosis, recurrence of the disease, or drug sensitivity will improve personalized medicine for the appropriate groups of patients. There are numerous biomarkers predicting drug sensitivity in the management of cancer but not all patients with such biomarkers show a positive therapeutic response [3]. A great number of small molecules act as regulators in several biological processes such as proliferation, differentiation, and programmed cell death. With the arrival of new therapeutic agents and the different responses displayed by patients, there will be a need for biomarkers to guide treatment selection.

The interest of laboratory and clinical investigations has been enormous which has resulted in voluminous publications (more than 150,000) related to proteomics and DNA microarrays [4]. The human genome contains more than two thousand mature micro RNA (miRNA) [5]. A single miRNA influences the expression of multiple genes, although a single gene may be influenced by several miRNAs. This situation generates a complex network and the analysis of miRNA panels is consistently more efficient in cancer studies than the analysis of a single small miRNA [6]. The large number of claimed biomarkers has been documented, but only fewer than one hundred have been validated for clinical practice [7-9].

Many pathological conditions are associated with changes in multiple molecular pathways, however, validation of this association is very challenging. In order to establish robust correlations between biomarkers and a human condition or response to therapy requires multidisciplinary expertise. Thus, the practicing oncologist should be informed about the latest developments in the area of biomarkers related

*Corresponding author: Nikolay V. Dimitrov, Professor Emeritus, Michigan State University, Division of Hematology and Oncology, Clinical Center, B-413, East Lansing, MI 48824, USA. Tel.: (517) 353-3128; Fax: 517-432-9471; Email: beth.moore@ht.msu.edu

Received 11 March 2017 Revised 06 June 2017 Accepted 14 June 2017 Published 24 June 2017

Citation: Isaac D, Elayoubi J, Almohammadi A, Dimitrov NV. Some aspects of cancer biomarkers and their clinical application in solid tumors - revisited.J Cancer Res Ther. 2017; 5(6):34-39. DOI: 10.14312/2052-4994.2017-7

Copyright: (c) 2017 Isaac D, et al. Published by NobleResearch Publishers. This is an open-access article distributed under the terms of the Creative Commons Attribution License, which permits unrestricted use, distribution and reproduction in any medium, provided the original author and source are credited. 
to prevention, diagnosis, and treatment of cancer and other pathological conditions. In this review, we present some points regarding the clinical utility of biomarkers in the management of the most common human cancers.

\section{Clinical utility of biomarkers in cancer}

Decreasing cancer mortality while minimizing the potential morbidity requires refined approaches to treatment and follow up of the patient's using novel noninvasive biomarkers. There are four important areas for clinical investigation which will facilitate the usefulness of biomarkers in the management of human cancer: 1. Optimal patient selection and specific type of biomarkers. 2. Collection and storage of the tissue/surrogate to be used; 3. Purity, sensitivity and interactions of the biomarker; 4. Validation and concordance between biomarkers.

It is generally accepted that cancer biomarkers indicate changes in the normal biological process related to growth, proliferation, differentiation and invasion. These changes could be intracellular or extracellular or be part of the cellular structure as a result of apoptosis or necrosis [10]. Biomarkers may have a potential value to predict the presence of minimal amount of tumor or to predict the future therapeutic resistance. In other cases, they may indicate a high risk condition for the development of malignancy.

A tumor marker's utility grading scale was introduced to facilitate discussion regarding the guidelines and their use in clinical practice [1]. Importantly, some biomarkers have been developed and validated for use in determination of a patient's risk of developing a malignancy and for the implementation of risk reducing strategies (example: BRCA mutational status). Other biomarkers have been used for prediction in determining response to a specific therapy. For example, somatic KRAS mutations are predictive of a poor response to anti-EGFR therapy [11]. Moreover, HER-2 mutational status is associated with response to anti-HER-2 based therapy in breast and gastric cancer (Figure 1).

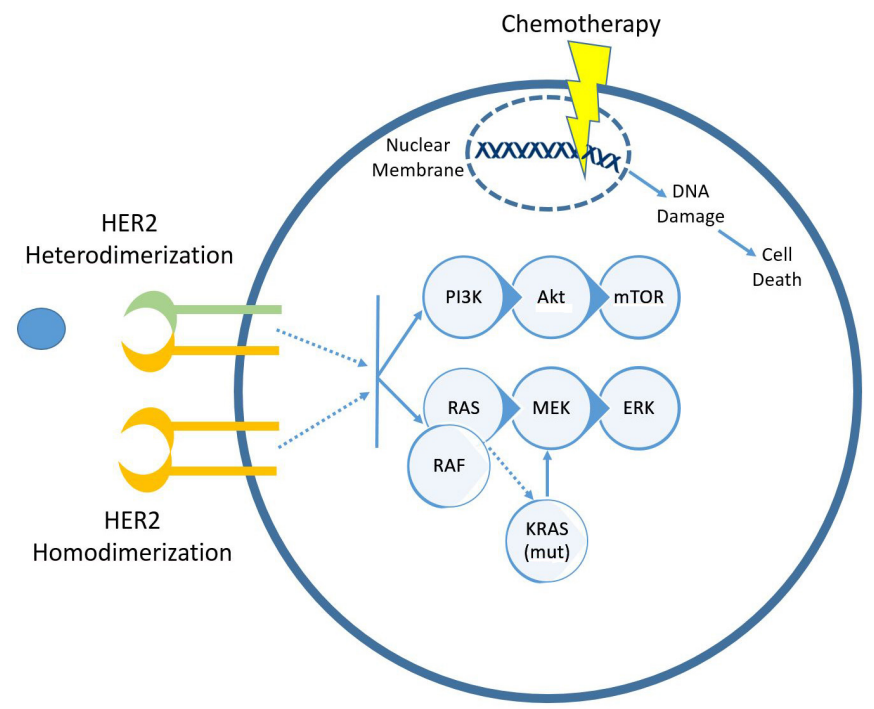

Figure 1 HER2 signaling pathways which are upregulated in a variety of cancers ultimately leading to increased cellular proliferation and survival. Target HER2 agents act to inhibit this signaling pathway and thus lead to cancer-cell death. On the other hand, chemotherapy acts to induce DNA damage resulting in cell death.
At present, targeted therapy includes small molecules and monoclonal antibodies which block special pathways related to tumor growth and carcinogenesis. Such molecules modify the function of proteins that regulate gene expression and other cellular functions. Therapeutic targets for these agents are present in some but not all tumor cells. Predictive biomarkers are needed to help identifying the subset of populations that may experience a favorable or unfavorable effect from a specific therapeutic intervention. A validated predictive biomarker is evaluated using in vitro companion diagnostic devices. Among the many potential predictive biomarkers is the protein survivin which is an inhibitor of apoptosis and plays crucial role in the regulation of cell division and cell cycle control. Survivin is strongly expressed in malignant tumors [12-14]. Survivin has been tested in both the in vivo and in vitro setting, however, information regarding it use in human cancers has been challenged. Using nanotechnology the determination of survivin will be easier and its use in cancer research and cancer treatment will meet the requirements for real time [12]. The oligonucleotide molecular beacons encoded for survivin mRNA have been proposed for the indirect detection of survivin expression [15]. Despite such advancements, a broad application of biomarkers in clinical practice is hampered by lack of specificity and generalizability to the population. As such, biomarker evaluation requires precise designation so as to ensure proper clinical implementation [3].

Currently, the most useful information is still obtained by histopathologic review. However, the search for alternative, non-invasive methods using biomarkers has been the focus of clinical research. One such method is the use of liquid biopsies which can help guide the clinician in appropriate diagnostic or therapeutic decisions [16, 17].

\section{Liquid biopsy}

Liquid biopsy is a concept that relies on information of circulating cells or cell components in the blood in order to obtain a diagnosis without the need for invasive approaches [16]. It has been shown that different metastatic sites can harbor different genomic aberrations and biopsy of one or two metastases may not be representative [18]. The biopsy of overt metastases is an invasive procedure limited to certain locations and not always acceptable in the clinic. An alternative approach is the analysis of blood samples for circulating tumor cells (CTCS) and/or circulating tumor DNA (ctDNA) obtained by liquid biopsy [19]. These biomarkers are remarkable because measuring cancer mutations offers exquisite specificity. The digital analysis of DNA sequence allows the high resolution detection of mutations. This method may play an important role in early detection of cancer, detections of minimal residual disease and monitoring of the evolution of molecular resistance $[8,19]$. A liquid biopsy can be used for characterization of the tumor $[10,20]$. Its non-invasive nature allows repeat sampling to monitor genetic changes over time without the need for a tissue biopsy. Thus, this approach can be used to track tumor evolution throughout treatment and enable dynamic adaptation of therapy (Figure 2).

\section{Genetic biomarkers}

Cancer cells are abundant signal transduction pathways 


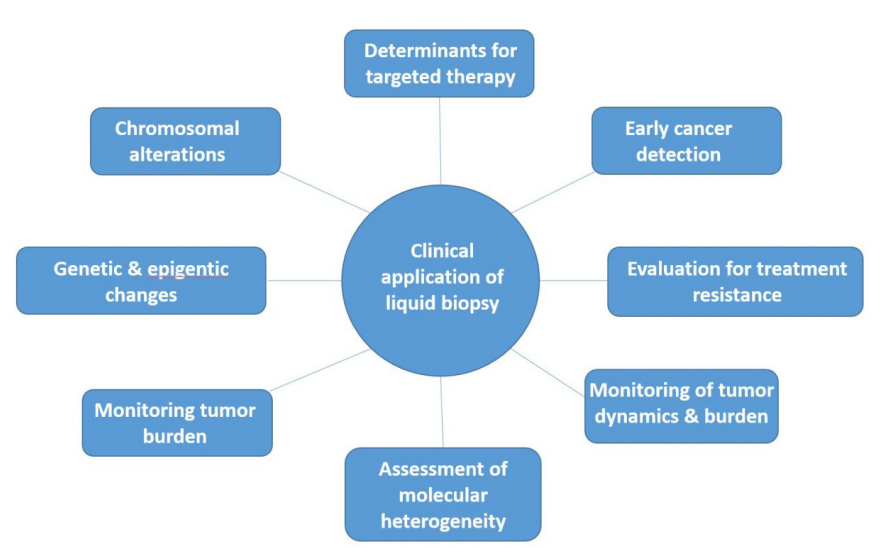

Figure 2 Current and potential clinical applications of liquid biopsies.

which respond to extracellular signals regulating downstream gene expressions [7]. A genetic marker is considered as an alteration in the germline copy of a cancer susceptibility gene that grants high risk for the development of malignancy. Germline alterations are congenital and heritable. They are associated with increased probability that the individuals carrying them will develop malignancy over their lifetime. Germline genetic alterations are present in all cells in the body in the DNA or as circulating proteins in the plasma or serum [21].

CTCs are tumor cells that are shed from solid tumors into the circulation and have been thought to harbor a potential for distant metastasis. There are multiple factors that impact the migration of CTCS; however, the impact of chemokine gradients (i.e. CXCR4, CCR4, etc) have been thought to play a critical role [22]. There have been a variety of methods developed for the detection of CTCS in the peripheral blood; however, these cells are present in very low concentrations in peripheral circulation and are often difficult to isolate given the extensive background of nucleated blood cells and erythrocytes. In addition, the halflife of CTCS is relatively short at around 1-2.4 hours [22]. Efforts to enhance the sensitivity of these methods have been previously investigated (i.e., reverse-transcription PCR assays) and offer a promising method for the clinical management of cancer.

The entry of ctDNA into the bloodstream is thought to originate from a cell following apoptosis, necrosis or both $[9,23,24]$. Late stage cancer patients have an increased level of cell free DNA (cfDNA) in plasma most of which is wild-type and believed to be from non-malignant cells and tumor stroma [25]. It has also been suggested that the mutant fraction of cfDNA is derived from necrotic neoplastic cells phagocytized by macrophages which release digested DNA [19]. The extensive background of wild-type DNA limits the ability of downstream analytical platforms to detect tumor-derived mutations. This represents technical challenges for the use of cfDNA in liquid biopsies. Cell free tumor DNA analyses are capable of examining the genetic or epigenetic changes that originate in tumor DNA. However, they cannot analyze the tumor RNA transcriptome or proteome [4].

Another substantial advantage of cfDNA is that it can be analyzed from bio-banked bio-fluids such as frozen plasma. However, the challenge for clinical and laboratory use is still present when using cfDNA to characterize the mutation status of a tumor. Besides the low copy number of mutant alleles, the median half-life of cfDNA in circulation ranges from 15 minutes to a few hours [26]. Delays in blood processing, blood storage temperature, agitation of the sample, and shipment can cause wild-type cfDNA release from lysed nucleated blood cells and affect allelic frequency [16]. For the same reason plasma is often preferred over serum because of the potential for cell lysis during blood coagulation [16]. Urinary and saliva cell free DNA may be a potential alternative to conventional primary tissue based analysis [18]. Using biomarker analysis through liquid biopsies might provide new insights into the biology and pathophysiology of the tumor. It is assumed that early detection and eradication of metastatic cells clearly has the potential to decrease cancer mortality. The studies on ctDNA combined with other genetic information may explain in a synergistic way why solid tumors develop resistance to targeted therapies [27].

All studies related to diagnosis, prognosis, drug resistance, and survival require the use of reliable biomarkers for follow up and validation of the results from liquid biopsies. In the field of cancers detection of cfDNA derived from tumors are designated as circulating tumor DNA (ctDNA) (Figure 2). Discriminating ctDNA from normal cfDNA is defined by presence of mutations. These somatic mutations are present only in the genomes of cancer cells or precancerous cells. This assures ctDNA biologic specificity as a biomarker. Unfortunately, ctDNA often represents a small fraction of the total cfDNA $(<1 \%)$ which is a challenge for this method [28]. That is why standard sequencing approaches can only detect tumor-derived mutant fragments in patients with heavy tumor burden. Introduction of new detection methods creates a wide array of practical clinical applications that are not possible with routine sequencing of tumor tissue (Figure 2).

Another area which requires more attention as a biomarker is the role of exosomes. Exosomes are small sized extracellular vesicles with a multi vesicular endosomal origin [29]. Due to their presence and stability in bodily fluids and the resemblance of their contents to parental cells, exosomes have a great potential to serve as a liquid biopsy tool for various cancers [30]. Tumorderived exosomes contain informative micro RNA involved in the interaction of cancer and microenvironment cells. They contribute to the tumor tissue remodeling of the microenvironment. There is a large body of evidence which has revealed that the tumor microenvironment is complex and cancer cells rely on it to sustain growth, invasion and survival [31]. Further evidence suggests that the exosomes are important signals in the cross-talk between various cell types and carry biological information consisting of transmembrane proteins, mRNAs, microRNAs as well as soluble and transmembrane proteins between the cells [32]. They are capable of being transported to local or distant anatomic locations where they can transduce signals or other information [33]. Exosomes have attracted the attention of many investigators and now are being evaluated as a potential biomarker [34]. More information regarding exosomes and their structure and function could be found in a review by Chlebowski and associates [35]. 


\section{Imaging biomarkers}

Tumors frequently reduce pyruvate to lactate rather than oxidize it in the tricarboxylic acid cycle (TCA/Kreb's cycle). It is important that this metabolic failure occurs even in the presence of oxygen (Warburg effect). A major function of TCA in tumors is to generate support for macromolecular biosynthesis. However, tumors can use fuels other than glucose for energy generation. The differences in metabolism between normal and tumor cells changes as the disease progresses. The response to treatment also makes the metabolic imaging technique an important tool for detecting and staging tumors and helps in guiding therapy for many patients. Accordingly, positron emission tomography (PET) scan could be defined as an imaging biomarker. Thus, PET scans with the glucose analogue 2-[18F]-2-fluoro-2-deoxy-D-glucose (FDG) is the most used metabolic imaging technique for tumor staging and assessment of therapeutic response [36].

\section{Biomarkers and common types of cancer}

\section{Prostate cancer}

Prostate cancer represents the third most common cause of cancer related death in men. The indolent clinical course represents a problem for early diagnosis, despite of use of some pre-therapeutic parameters such as Gleason grade and tumor extent on biopsies, preoperative prostatespecific antigen (PSA) and clinical stage. However, the availability of clinical data is not sufficient for optimal individual therapeutic decisions. There is an obvious need for reliable prognostic parameter for use as part of personalized medicine. A variety of methods including genomic alterations have been proposed, but the search for subtle marker is still clinically experimental [24].

\section{Lung cancer}

Despite extensive clinical and laboratorystudies, lung cancer detection using a single biomarker remains unsuccessful due to the low sensitivity, specificity, and reproducibility. Lung cancers are very heterogeneous histologically which is reflected at the molecular level and represent a huge problem during performance and interpretation of the clinical tests. It limits the significance of the single test and raises the questions regarding multiple testing. The search for tissue-specific biomarkers has been intensifying during the last two decades and still attracts the attention of many cancer researchers. Many of the published studies on "lung cancer biomarkers" is of limited clinical usage. Validation of the lung cancer biomarkers is very important because recruitment of patients for participation in well-designed clinical trials has become a universal problem.

The follow up of patients with lung cancer using specific biomarkers is important. The use of liquid biopsy for these patients will facilitate the validation of clinical trials. The introduction of plasma and urine genotyping may fortify the identification of mutations in non-small cell lung cancer and effectively improved the treatment outlook [37, 38]. The introduction of checkpoints as biomarkers and the role of the immune checkpoint inhibitors in lung cancer is an area of active research.

\section{Colorectal cancer}

Besides the presence of strong evidence supporting the efficacy of colonoscopy, this procedure is limited by its invasiveness, expense, and suboptimal patient compliance. Colorectal cancer related mortality has declined partly due to the early detection through robust screening including colonoscopy. Accordingly, efforts are underway to develop more specific biomarker assays as means of risk and early detection of advanced colon polyps. A variety of fecal based and blood based biomarkers have been proposed $[11,39]$. It is generally accepted that patients with newly diagnosed metastatic colon cancer should receive KRAS, RAS and BRAF testing and be assessed for microsatellite instability. Currently, the methylated DNA in the blood is an established blood biomarker with 69\% sensitivity and $86 \%$ specificity for distinguishing patients with colorectal cancer from healthy individuals [40]. It is conceivable that small tumors obtained by colonoscopy are potential candidates for cure if successful biomarker guided therapy is applied. The currently available blood based biomarkers assays are not appropriate as colon polyp detection method.

\section{Breast cancer}

Evaluation of breast cancer biomarkers is a standard procedure in the medical oncology practice. Substantial progress has been made with the use of hormone receptors and HER-2 as predictors of treatment response. Other proteins can serve as predictive biomarkers for trastuzumab resistance [7, 41]. The role of androgen and estrogen dual expression is another area for future investigation [42]. Trastuzumab is one of the first examples of personalized cancer therapy where improved clinical outcomes are reported in patients with HER-2 positive breast tumors. The biomarker is found in several other solid tumors [43]. Breast cancer and biomarkers related to tumor growth, metastasis, response to therapy and tumor resistance are widely reported [7, 44]. However, it is crucial to quickly identify new biomarkers with the potential to enhance early diagnosis and to predict a patient's prognosis, development of drug resistance and treatment choice. The introduction of liquid biopsy and new valuable biomarkers in primary breast cancer will offer the opportunity to challenge the historical approach to management of this disease. It could lead to the development clinical trials of novel prolonged adjuvant therapy designed with combined or sequential molecular and biological therapies with liquid biopsy based molecular monitoring of micro-metastatic disease.

\section{Use of liquid biopsy sources}

The most important sources of liquid biopsies are serum and plasma which are easy to obtain and allow frequent sampling, transportation and storage. Plasma is often preferred over serum because of potential problems in blood processing and cell lysis during blood coagulation. Other sources such as sputum, saliva, bronchoalveolar lavage, nipple discharge and pleural or peritoneal effusions have been reported [45]. However, their utility in the clinical use is limited due to the purity of sample challenges and analysis.

Another source for a variety of studies are the peripheral blood lymphocytes (PBL), which are easy to obtain and to separate as $90-100 \%$ pure cellular samples. These cells allow further morphological, metabolic, or genetic studies. 
The PBL are T cells which infiltrate the tumor tissue and after communication with the abnormal cells, re-enter the circulation where they could be collected for a variety of laboratory studies. Information regarding the designation of biomarkers is needed because the samples contain various lymphocytes (TILs, NK cells, etc). Our laboratory has successfully used these lymphocytes for metabolic studies $[46,47]$. With the fast return of immunotherapy as a therapeutic challenge, the PBL could serve as a surrogate tissue biomarker in various solid tumors.

Lymphocytes could be reliable biomarkers [46, 47]. It is generally accepted that interaction between lymphocytes and cancer cells is an essential component in the defense against the growth of neoplastic cells in the human body [4850]. The lymphocytes are able to adhere and to penetrate into the tumor and remain inside. In addition, they are capable of recirculating. The contact between the tumor cell is probably mediated by a sensation of the lymphocyte to tumor-specific antigens [51]. The ERK group of MAPKs are known to play an important role in the transduction of mitogenic signals that culminate in cell growth and proliferation [52]. This activity has also been demonstrated in lymphocytes $[46,52]$. Thus the lymphocyte could be used as surrogate tissue for development of biomarkers. In addition, the metabolic change in peripheral lymphocytes are targets for development of new biomarkers.

\section{Anticancer drugs and cancer biomarkers}

One of the most important uses of biomarkers is to guide decisions on systemic therapy for patients with newly diagnosed metastatic cancers and with time moving to the adjuvant setting. To facilitate future updates in a rapidly developing and growing field, multidisciplinary groups (such as ASCO committees) will be needed to determine guidelines for use of biomarkers to guide or influence decisions on systemic therapy. There is growing interest for the development of molecularly targeted agents that block or stimulate specific signaling pathways of cancer cells [53]. These targeted agents have diverse mechanisms of actions such as inducing programmed cell death (apoptosis) of cancer cells, blocking specific enzymes, and growth factor receptors involved in cancer cell proliferation or modifying the function of proteins that regulate gene expression and variety of other cellular function. By acting on specific oncogenic proteins, these targeted therapies hold promise for improved therapeutic outcome [53].

Unfortunately, there is vast heterogeneity in tumors and patients. As such, predictive biomarkers are needed to identify subsets of populations that are most likely to experience a favorable or unfavorable therapeutic effect [54]. Signaling components of human epidermal growth factor receptor 2 (HER-2), epidermal growth factor receptor (EGFR) and programmed death receptor-1 (PD-1) are good examples of the successful development of molecular biomarker-driven cancer therapy. Expanding the knowledge of predictive biomarkers will help to identify new surrogate markers which could be used in the clinical investigation for early readouts of drug efficacy. Biomarkers characterization and evaluation can help usher in a new era of personalized oncology.

\section{Discussion}

The recent advances in technology and biology have improved the identification, detection and verification of biomarkers and thus, improved our understanding of human cancers. Although many biomarkers have been developed, validated and tested in clinical trials mortality of several cancers remain high. Reliable and robust noninvasive diagnostic and screening biomarkers that are conveniently detectable in the peripheral blood are still in demand.

\section{Conclusion}

There is an increasing need for the development and validation of novel biomarkers in human cancers. Unfortunately, the development of biomarkers has been fraught with limited reproducibility in clinical studies. With the development of novel immunotherapies the need for biomarkers to predict tumor sensitivity and monitor therapeutic response is necessary. Perhaps one of the most promising strategies for novel biomarker development is via the liquid biopsy. The liquid biopsy may be defined as a test with analytical sensitivity which can detect single tumor cells. This biopsy should be carried out in real time and allowed characterization of normal and cancerous tissue by using reliable biomarkers. It has been shown that metastatic cells may have phenotypic and genomic characteristics that are distinct from those of the primary tumor. Thus, the metastatic cells may gain additional genomic characteristics over time and develop independently from the primary tumor. The role of ctDNA should be further investigated. In addition, the role of the PBL offers a promising strategy to further define the morphologic, metabolic and genetic characteristics of cancer cells and should be evaluated as a potential biomarker for future development.

\section{Funding}

Research Funding - Michigan State University Department of Medicine.

\section{Acknowledgements}

The valuable technical assistance of Ms. Beth Moore is highly appreciated.

\section{Conflicts of interests}

Authors declare no conflicts of interests.

\section{References}

[1] Hayes DF, Bast RC, Desch $C E$, Fritsche $H$, Kemeny $N E$, et al. Tumor marker utility grading system: a framework to evaluate clinical utility of tumor markers. J Natl Cancer Inst. 1996; 88(20):1456-1466.

[2] Hayes DF. When is a tumor marker ready for prime time? J Clin Onc. 1997; 206-212.

[3] Henry NL, Hayes DF. Cancer biomarkers. Molecular Oncology. 2012; 6(2):140-146.

[4] Poste G. Bring on the biomarkers. Nature. 2011; 469(7329):156-157.

[5] Friedman RC, Farh KK, Burge CB, Bartel DP. Most mammalian mRNAs are conserved targets of microRNAs. Genome Res. 2009; 19(1):92105.

[6] Izzotti Al, Carozzo S, Pulliero A, Zhabayeva D, Ravetti JL, et al. Extracellular microRNA in liquid biopsy: applicability in cancer diagnosis and prevention. Am J Cancer Res. 2016; 6(7):1461-1493. 
[7] Murtaza M, Dawson SJ, Tsui DW, Gale D, Forshew T, et al. Non-invasive analysis of acquired resistance to cancer therapy by sequencing plasma DNA. Nature. 2013; 497(7447):208-212.

[8] Cooper SJ, Tun HW, Roper SM, Kim Y, Kislinger T, et al. Current status of biomarker discovery in human clear cell renal cell carcinoma. J Mol Biomark Diagn. 2012; S2:005.

[9] Karachaliou N, Mayo-de-las-casas C, Molina-Vila MA, Rosell R. Real time liquid biopsies become a reality in cancer treatment. Ann Transl Med. 2015; 3(3):36.

[10] Diehl F, Schmidt K, Choiti MA, Romans K, Goodman S, et al. Circulating mutant DNA to assess tumor dynamics. Nat Med. 2008; 14(9):985990.

[11] Allegra CJ, Jessup JM, Somerfield MR, Hamilton SR, Hammond EH, et al. American society of clinical oncology provisional clinical opinion: testing for KRAS gene mutations in patients with metastatic colorectal carcinoma to predict response to anti-epidermal growth factor receptor monoclonal antibody therapy. J Clin Oncol. 2009; 27(12):2091-2096.

[12] Stobiecka M, Dworakowska B, Jakiela S, Lukasiak A, Chalupa A, et al. Sensing of survivin mRNA in malignant astrocytes using graphene oxide nanocarrier-supported oligonucleotide beacons. Sens Actuators B Chem. 2016; 235:136-145.

[13] Altieri DC. The molecular basis and potential role of survivin in cancer diagnosis and therapy. Trends Mol Med. 2001; 7(12):542-547.

[14] Blanc-Brude OP, Mesri M, Wall NR, Plescia J, Dohi T, et al. Therapeutic targeting of the surviving pathway in cancer. Clin Canc Res. 2003; 9(7):2683-2692.

[15] Peng XH, Cao ZH, Xia JT, Carlson GW, Lewis MM, et al. Real-time detection of gene expression in cancer cells using molecular beacon imagining: New strategies for cancer research. Cancer Res. 2005; 65(5):1909-1917.

[16] Crowley E, Di Nicolantonio F, Loupakis F, Bardelli A. Liquid biopsy: Monitoring cancer-genetics in the blood. Nature. 2013; 10(8):472484.

[17] Bellassai N, Giuseppe S. Biosensors for liquid biopsy: Circulating nucleic acids to diagnose and treat cancer. Anal Bioanal Chem. 2016; 408(26):7255-7246.

[18] Gerlinger M, Rowan AJ, Horswell S, Larkin J, Endesfelder D, et al. Intratumor heterogeneity and branched evolution revealed by multiregion sequencing. N Engl J Med. 2012; 366(10):883-892.

[19] Parsons HA, Beaver JA, Park BH. Circulating plasma tumor DNA. Adv Exp Med Biol. 2016; 882:259-276.

[20] Turajlic S, Swanton C. Tracking tumor evolution through liquid biopsy. Nat Rev Clin Oncol. 2015; 12(10):565-566.

[21] Shattuck-Eidens D, Oliphant A, McClure M, McBride C, Gupte J, et al. BRCA1 sequence analysis in women at high risk for susceptibility mutations: Risk factor analysis and implications for genetic testing. JAMA. 1997; 278(15):1242-1250.

[22] Alix-Panabieres C, Pantel K. Clinical applications of circulating tumor cells and circulating tumor DNA as liquid biopsy. Cancer Discov. 2016; 6(5):479-491.

[23] Goebel G, Zitt M, Muller HM. Circulating nucleic acids in plasma or serum (CNAPs) as prognostic and predictive markers in patients with solid neoplasias. Dis Markers. 2005; 21(3):105-120.

[24] Gormally E, Caboux E, Vineis P, Hainaut P. Circulating free DNA in plasma or serum as biomarker of carcinogenesis: Practical aspects and biological significant. Mutat Res. 2007; 635(2-3):105-117.

[25] Todenhöfer T, Hennenlotter J, Kühs U, Tews V, Gakis G, et al. Influence of urinary tract instrumentation and inflammation on the performance of urine markers for the detection of bladder cancer. Urology. 2012; 79(3):620-624

[26] Kamat AM, Hegarty PK, Gee JR, Clark PE, Svatek RS, et al. ICUD-EAU international consultation on bladder cancer 2012: Screening, diagnosis and molecular markers. Eur Urol. 2013; 63(1):4-15.

[27] Misale S, Yaeger R, Hobor S, Scala E, Janakiraman M, et al. Emergence of KRAS mutations and acquired resistance to anti-EGFR therapy in colorectal cancer. Nature. 2012; 486(7404):532-536.

[28] Jung K, Fleischhaker M, Rabien A. Cell free DNA in the blood as a solid tumor biomarker - a critical appraisal of the literature. Clin Chim Acta. 2010; 411(21-22):1611-1624.

[29] Tkach M, Thery C. Communication by extracellular vesicles: Where we are and where we need to go. Cell. 2016; 164(6):1226-1232.

[30] Rak J. Extracellular vesicles - Biomarkers and effectors of the cellular interactome in cancer. Front Pharmacol. 2013; 4:21.

[31] Quail DF, Joyce JA. Microenvironmental regulation of tumor progression and metastasis. Nat Med. 2013; 19(11):1423-1437.

[32] Pefanis E, Wang J, Rothschild G, Lim J, Kazadi D, et al. RNA exosome regulated long non-coding RNA transcription controls super-enhancer activity. Cell. 2015; 161(4):774-789.

[33] Li L, Li C, Wang S, Wang Z, Jiang J, et al. Exosomes derived from hypoxic oral squamous cell carcinoma cells deliver miR-21 to normoxic cells to elicit prometastatic phenotype. Cancer Res. 2016; 76(7):1770-1780.

[34] Monzo M, Santasusagna S, Moreno I, Martinez F, Hernandez R, et al. Exosomal microRNAs isolated from plasma of mesenteric veins linked to liver metastases in resected patients with colon cancer. Oncotarget. 2017; 8(19):30859-30869.

[35] Chlebowski A, Lubas M, Jensen TH, Dziembowski A. RNA decay machines: The exosome. Biochim Biophys Acta. 2013; 1829(6-7):552560.

[36] Timm KN, Kennedy BWC, Brindle KM. Imaging tumor metabolism to assess disease progression and treatment response. Clin Cancer Res. 2016; 22(21):5196-5203.

[37] Chen S, Zhao J, Cui L, Liu Y. Urinary circulating DNA detection for dynamic tracking of EGFR mutations for NSCLC patients treated with EGFR-TKIs. Clin TransI Oncol. 2017; 19(3):332-340.

[38] Chabon JJ, Simmons AD, Lovejoy AF, Esfahani MS, Newman AM, et al. Circulating tumour DNA profiling reveals heterogeneity of EGFR inhibitor resistance mechanisms in lung cancer patients. Nat Commun. 2016; 7:11815.

[39] Williams CD, Grady WM, Zullig LL. Use of NCCN guidelines, other guidelines and biomarkers for colorectal cancer screening. J Natl Compr Canc Netw. 2016; 14(11):1479-1485.

[40] Lofton-Day C, Model F, Devos T, Tetzner R, Distler J, et al. DNA methylation biomarkers for blood-based colorectal cancer screening. Clin Chem. 2008; 54(2):414-423.

[41] Berns K, Sonnenbuck A, Gennissen A, Brohee E, Hijmans M, et al. Loss of ARID1A activates ANXA1, which serves as a predictive biomarker for trastuzumab resistance. Clinical Cancer Res. 2016; 22(21):5238-5248.

[42] Li W, O'shaughnessy, Hayes D, Campone M, Bondarenko I, et al. Biomarker association with efficacy of abiraterone acetate and exemestane in postmenopausal patients with estrogen receptor positive metastatic breast cancer. Clin Cancer Res. 2016; 22(24):60026009.

[43] Baniak N, Senger JL, Ahmed S, Kanthan SC, Kanthan R. Gastric biomarkers: A global review. World J Surg Oncol. 2016; 14(1):212-226.

[44] Van Poznak C, Somerfield MR, Bast RC, Cristofanilli M, Goetz MP, et al. Use of biomarkers to guide decisions on systemic therapy for women with metastatic breast cancer: American society of clinical oncology practice guideline. J Clin Oncol. 2015; 33(24):2695-2704.

[45] I H, Cho JY. Lung Cancer Biomarkers. Adv Clin Chem. 2015; 72:107170.

[46] Dimitrov NV, Ellegaard J. Elevated lymphocyte adenosine phosphatase activity in patients with gastrointestinal carcinoma. N Engl J Med. 1972; 286(7):353-355.

[47] Madhukar BV, Dimitrov NV, Meyer-Leece C, Contreras ML, Crowell $J$. Inhibition of mitogen-activated protein kinase activity of human lymphocytes after oral administration of Oltipraz. Mol Cancer Ther. 2002; 1(12):1125-1128.

[48] Meuwissen HJ, Stutman O, Good RA. Functions of the lymphocytes. Semin Hematol. 1969; 6(1):28-66.

[49] Humble JG, Jayne WHW, Pulvertaft RJV. Biological interaction between lymphocytes and other cells. Br J Haematol. 1956; 2(3):283-294.

[50] Craddok CG, Longmire R, McMillin R. Lymphocytes and the immune response. N Engl J Med. 2971; 285(6):324-331.

[51] Alexander P, Delorme EJ, Hall JG. The effect of lymphoid cells from the lymph of specifically immunized sheep on the growth of primary sarcomata in rats. Lancet. 1966; 1:1186-1189.

[52] Gudermann T. Multiple pathways of ERK activation by $G$ protein coupled-receptors. Novartis Found Symp. 2001; 239:68-79.

[53] Twomey JD, Brahme NN, Zhang B. Drug-biomarker co-development in oncology - 20 years and counting. Drug Resist Updat. 2017; 30:48-62.

[54] Nalejska E, Maczyriska E, Lewandowska MA. Prognostic and predictive biomarkers: Tools in personalized oncology. Mol Diagn Ther. 2014; 18(3):273-284. 